\title{
Prion variant pathogenicity through large-scale population sequencing
}

Familial studies have identified many gene variants that underlie monogenic Mendelian disorders; however, for currently asymptomatic individuals in the general population who harbour these variants, the actual future disease risk is often poorly defined. A new study uses large-scale population sequencing data to characterize the pathogenicity of prion disease variants, for improved predictive value in genetic counselling.

The penetrance of disease-associated genetic variants is the proportion of individuals harbouring that variant who will actually be affected by the associated disease. As disease-associated variants are frequently identified and characterized in the context of affected family pedigrees, this can lead to ascertainment bias, in that the pathogenicity of the variant allele is overestimated. Therefore, surveys of the prevalence of those variants in large cohorts of asymptomatic individuals are required to determine the true penetrance of the variant, which enables more accurate estimates of future disease risk in healthy people who discover from genetic analyses that they harbour these variants.

Minikel et al. studied variants in the PRNP gene, which encodes prion protein ( $\mathrm{PrP}$ ). Misfolded $\mathrm{PrP}$ is responsible for prion diseases, which comprise various rare diseases such as Creutzfeldt-Jakob disease. Despite the media attention paid to cases of prion diseases that are linked to transmission of misfolded PrP through dietary or surgical routes, these represent only a few cases, whereas the vast majority of cases are either sporadic ( 85\%) or linked to inheritance of disease-associated PRNP variants ( 15\%). As PRNP variants are known to be rare in the overall population, the authors required large cohorts in order to assess penetrance. They analysed the frequencies of PRNP variants in 16,025 prion disease cases, in addition to 2 control cohorts: 60,706 individual exome sequences from the Exome Aggregation Consortium (ExAC) data set and 531,575 individuals genotyped by the 23 andMe direct-to-consumer genetic testing service.

The penetrance estimates allowed the authors to group PRNP variants into different pathogenic classes. Some amino-acid-substitution (missense) variants, such as E200K, were highly penetrant, based on their almost complete absence in control individuals and previous strong genetic and functional evidence linking them to prion disease. By contrast, other variants such as E196A seem to be benign, as there were no meaningful differences in frequencies between cases and controls. Between these extremes were variants

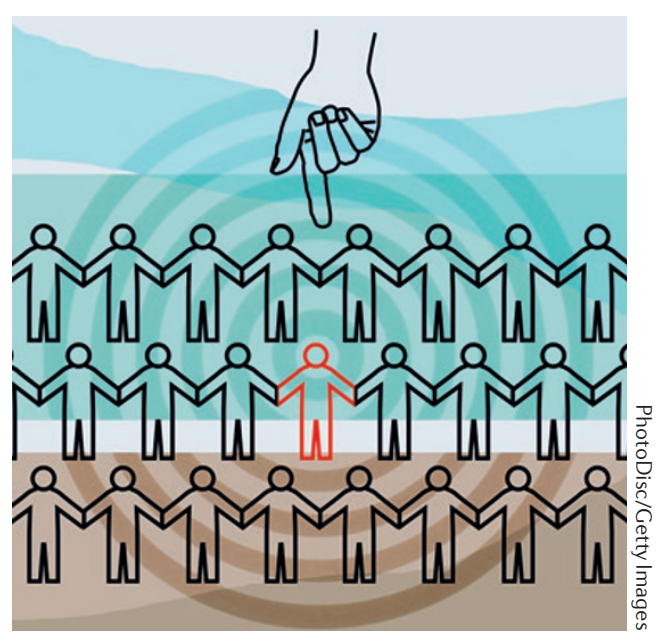

such as $\mathrm{V} 180 \mathrm{l}$, which had incomplete penetrance; despite increasing disease risk substantially ( $\sim 50$-fold), the overall rarity of prion diseases means that individuals harbouring this variant have a lifetime disease risk of only $\sim 1 \%$. Beyond missense variants, the team also characterized protein-truncating PRNP variants, finding that the expected gain-of-function pathogenicity seems to be conferred only by carboxy-terminal truncations that leave most of the protein intact.

Despite the large size of the study, the authors reported confident penetrance estimates and pathogenicity classifications for only 10 of 63 reportedly pathogenic PRNP variants, highlighting the challenges of interpreting rare alleles of unknown clinical significance. Still, for individuals harbouring any of these ten variants, this study provides greater certainty regarding disease risk.

In an accompanying perspective piece, Lebo, Sutti and Green outline the motivation and impetus behind the study. The first two authors of the study, Eric Minikel and Sonia Vallabh, are a husband and wife team who pursued scientific research careers after being motivated by a personal desire to understand the medical implications of a PRNP mutation within Vallabh's family.

Such a study highlights the power of curiosity-driven individuals to advance disease genetics research, both as drivers of research directions and as willing participants in large-scale, collaborative genetic analysis projects.

Darren J. Burgess

ORIGINAL ARTICLES Minikel, E. V. et al. Quantifying prion disease penetrance using large population control cohorts. Sci. Transl. Med. 8, 322 ra9 (2016) | Lebo, M. S., Sutti, S. \& Green, R. C. "Big Data” gets personal. Sci. Transl. Med. 8, 322fs3 (2016) 